Full length article

\title{
Lower extremity gait kinematics outcomes after knee replacement demonstrate arthroplasty-specific differences between unicondylar and total knee arthroplasty: A pilot study
}

\author{
Henri De Vroey ${ }^{\mathrm{a}, *}$, Filip Staes ${ }^{\mathrm{b}}$, Evie Vereecke ${ }^{\mathrm{c}}$, Jos Vanrenterghem ${ }^{\mathrm{b}}$, Jan Deklerck ${ }^{\mathrm{a}}$, \\ Geert Van Damme ${ }^{\mathrm{e}}$, Hans Hallez ${ }^{\mathrm{d}}$, Kurt Claeys ${ }^{\mathrm{a}, \mathrm{b}}$ \\ ${ }^{a}$ Department of Rehabilitation Sciences, KU Leuven Campus Bruges, Spoorwegstraat 12, 8200 Bruges, Belgium \\ ${ }^{\mathrm{b}}$ Department of Rehabilitation Sciences, KU Leuven, Tervuursevest 101, 3001 Heverlee, Belgium \\ ${ }^{\mathrm{c}}$ Department of Development and Regeneration, KULAK, Etienne Sabbelaan 53, 8500 Kortrijk, Belgium \\ ${ }^{\mathrm{d}}$ Department of Computer Science, KU Leuven Campus Bruges, Spoorwegstraat 12, 8200 Bruges, Belgium \\ ${ }^{\mathrm{e}}$ Department of Orthopaedic Surgery, AZ Sint-Lucas, Sint-Lucaslaan 29, 8310 Bruges, Belgium
}

\section{A R T I C L E I N F O}

\section{Keywords:}

Gait analysis

Kinematics

Knee replacement

Total knee arthroplasty

Unicondylar knee arthroplasty

\begin{abstract}
A B S T R A C T
Background: The aim of the present study is to compare sagittal gait kinematics of ankle, knee and hip joints between subjects with unicondylar and total knee arthroplasty and age matched healthy controls. Since unicondylar knee replacement is a less invasive procedure, which more closely preserves knee joint anatomy, we hypothesized that one year post unicondylar knee arthroplasty patients would demonstrate more normal gait patterns than patients with total knee arthroplasty.

Research question: Do unicondylar and total knee arthroplasty patients display similar gait kinematics one year after surgery?

Methods: Fourteen subjects (8 posterior stabilized and 6 medial unicondylar knee replacements) that were one year post surgery, and 6 healthy control subjects underwent a 3D gait analysis and a physical examination (range of motion, muscle strength). Statistical parametric mapping was used to compare gait kinematics of the lower limbs between groups. Additionally, differences in peak angles and clinical outcomes were assessed using a oneway ANOVA between subjects analysis.

Results: Both knee replacement groups showed reduced knee flexion range of motion and reduced muscle strength at the operated leg compared to the control group. Subjects with TKA demonstrated reduced knee flexion at loading response and midstance of the gait cycle. Both UKA and TKA demonstrated significantly less knee flexion during swing.

Significance: The results of this study demonstrate arthroplasty-specific differences in muscle strength, range of motion and gait kinematics of the lower limb one year after knee surgery. Future planning of post-surgery follow-up should addresses these arthroplasty-specific weaknesses and gait deviations.
\end{abstract}

\section{Introduction}

Knee replacement surgery is a commonly used treatment option for the management of end-stage knee osteoarthritis. Although this procedure aims to improve functionality and mobility, its primary indication is pain $[15,24]$. Over the past decade, a strong increase in knee replacement procedures has been observed in Western countries $[3,43]$. Additionally, epidemiological studies have reported that up to $30 \%$ of knee replacement patients present knee joint degeneration, which is limited to the medial or lateral tibiofemoral compartment [27]. Therefore, implant designs have been developed and fine-tuned in order to meet the mechanical and anatomical requirements of these patients. For instance, unicondylar knee arthroplasty (UKA) only replaces the compartment (medial or lateral) that demonstrates the most degenerative lesions. Moreover, both cruciate ligaments are preserved in a UKA procedure, which is believed to be beneficial for joint stability

\footnotetext{
* Corresponding author.

E-mail addresses: henri.devroey@kuleuven.be (H. De Vroey), filip.staes@kuleuven.be (F. Staes), vereecke@kuleuven.be (E. Vereecke), jos.vanrenterghem@kuleuven.be (J. Vanrenterghem), jan.deklerck@kuleuven.be (J. Deklerck), geert.vandamme@stlucas.be (G. Van Damme), hans.hallez@kuleuven.be (H. Hallez), kurt.claeys@kuleuven.be (K. Claeys).
} 
and proprioception [37]. The latter is in contrast with the majority of total knee arthroplasty (TKA) implants (e.g. Posterior stabilized TKA), which replace all articular surfaces at the knee joint and during which the cruciate ligaments are removed. Furthermore, UKA has been associated with less post-operative complications (blood loss, infection) and a shorter hospital stay compared to TKA $[7,44]$. However, UKA implants are more frequently revised then TKA. Nevertheless, UKA procedures are gaining popularity in the management of unicompartmental knee osteoarthrosis $[17,35]$.

Based on patient-reported measurement outcomes and clinical testing, good to excellent results have been reported for both the UKA and TKA designs [15]. However, despite the well-documented success of both implants, up to $30 \%$ of patients report difficulties when performing activities of daily living (e.g. walking) after surgery [5]. Previous research has demonstrated that questionnaire-based assessments lack sensitivity to capture the actual functional ability of patients, which may be the origin for the conflicting results mentioned above [21]. Furthermore, since younger patients typically have higher demands regarding the functioning of their implant, a thorough motion analysis may provide clinicians and researchers with valuable information that can potentially enhance rehabilitation and facilitate recovery after a knee replacement surgery [41].

Three-dimensional motion analysis provides an in depth evaluation of joint kinematics during dynamic tasks and can be related to the actual functioning of patients $[4,33]$. Knee kinematics during gait have been studied widely in patients with TKA and UKA [29,30]. However, since gait is a complex motor task that requires coordinated motion of the entire lower limb [22], one would expect previous authors to have investigated ankle, knee and hip joint kinematics. Remarkably, only few studies included a multi-joint approach, also called a lower limb kinematic chain approach, as they only focused on knee joint kinematics or peak knee joint angles [20,25]. Yet, a peak angle analysis does not yield any information regarding the resulting kinematical curve. Therefore, statistical parametric mapping (SPM) was used in the present study as a time series analysis tool, which made it possible to identify significant differences between kinematical curves produced by the different groups [36]. Besides kinematics, the spatiotemporal gait parameters also yield important clinical information, as they have been related to several functional conditions (e.g. risk of falling and frailty syndrome) $[10,38]$. Again, few studies have compared spatiotemporal variables between UKA, TKA and healthy control subjects [26]. Finally, it is of interest to assess patients at least one year after their knee replacement, as it has been demonstrated in severe knee pathology that significant progress can still be made up to this point [21]. However, no evidence comparing the aforementioned variables between UKA, TKA and healthy controls at one year post knee replacement surgery could be found.

Therefore, the general aim of this study is to gain more insight into the gait kinematics of the entire lower limb, one year after UKA or TKA surgery. A first specific aim is to compare the kinematics and spatiotemporal parameters of gait between UKA, TKA and healthy subjects. A second specific aim is to compare the clinical outcome after UKA or TKA with a healthy control group.

\section{Methods}

\subsection{Subjects}

Twenty subjects (6 UKA, 8 TKA and 6 Healthy) were recruited to participate in this study. Participants' demographics can be found in Table 1. All subjects were recruited at the orthopedic ward of the AZ Sint-Lucas hospital in Bruges. Subjects were excluded if they had any comorbidity affecting gait such as orthopedic surgery, previous trauma to the lower limbs or a history of neurologic or systemic disease. A written informed consent was obtained from all individual participants. All procedures were in accordance with the ethical standards of the
Table 1

Participants' demographics.

\begin{tabular}{lllll}
\hline & UKA $(\mathrm{n}=6)$ & TKA $(\mathrm{n}=8)$ & Healthy $(\mathrm{n}=6)$ & $\mathrm{P}$ \\
Mean $( \pm \mathrm{SD})$ & Mean $( \pm \mathrm{SD})$ & Mean $( \pm \mathrm{SD})$ & \\
\hline Gender & $\mathrm{M}=4 / \mathrm{F}=2$ & $\mathrm{M}=2 / \mathrm{F}=6$ & $\mathrm{M}=5 / \mathrm{F}=1$ & \\
Age (years) & $66.50(9.71)$ & $64.50(3.85)$ & $63.33(3.61)$ & n.s. \\
Weight $(\mathrm{kg})$ & $86.50(18.85)$ & $89.00(17.74)$ & $73.80(5.88)$ & n.s. \\
Height $(\mathrm{m})$ & $1.69(0.14)$ & $1.65(0.06)$ & $1.68(0.06)$ & n.s. \\
BMI $\left(\mathrm{kg} / \mathrm{m}^{2}\right)$ & $30.08(4.33)$ & $32.99(7.14)$ & $26.03(1.55)$ & n.s. \\
$\begin{array}{l}\text { OKS-score } \\
\text { Age of the }\end{array}$ & $44.00(2.76)$ & $41.50(6.19)$ & $\mathrm{NA}$ & n.s. \\
$\quad$ arthroplasty & $452.17(90.47)$ & $465.13(94.97)$ & $\mathrm{NA}$ & n.s. \\
$\quad$ & & & & \\
\hline
\end{tabular}

UKA: Unicondylar Knee Arthroplasty; TKA: Total Knee Arthroplasty; M: male; F: Female; SD: Standard Deviation; OKS: Oxford Knee Score.

institutional research committee of KU Leuven and with the Helsinki declaration.

\subsection{Clinical examination}

An experienced physiotherapist performed a clinical examination. Active sagittal range of motion (RoM) of the knee and ankle joint was evaluated using a standard goniometer [6]. Measurements of the ankle joint were completed with the knee in an extended position. Muscle strength was evaluated by means of a break test [32], using a hand-held dynamometer (MicroFET2, Hoggan Health Industries, Salt Lake City, US). Subjects performed hip abduction, knee extension, knee flexion and hip extension against manual resistance of the researcher to assess the gluteus medius, quadriceps, hamstrings and gluteus maximus respectively. Muscle force values were corrected for height and weight of the subjects based on the formula described by Krause et al. [23]:

Corrected muscle strength value

$$
=\frac{\text { Actual muscle strength }(\mathrm{N} / \mathrm{m})}{[\text { subject height }(\mathrm{cm}) * \text { subject mass }(\mathrm{Kg})]} * 100
$$

Perceived functionality was investigated with the Oxford 12-item Knee Score (OKS) questionnaire [12].

\subsection{Gait analysis}

Data were collected from each subject as they walked barefoot in a straight line along a $6 \mathrm{~m}$ long walk way. Each subject performed three walking trials at a self-selected speed. Marker data were gathered at a sampling frequency of $120 \mathrm{~Hz}$ using an Optitrack flex 13 (six cameras) system (Naturalpoint, Corvalis, US). Reflective markers (44) were placed according to the lower limb and trunk model described by Vanrenterghem et al. [42]. Anatomical markers were rigidly secured at the following locations: cervical vertebra C7, sternum, xiphoid process, thoracic vertebraT8, both posterior superior iliac spines, both anterior superior iliac spines, both iliac crests, both acromions, both greater trochanter, the medial and lateral knee epicondyle, the medial and lateral malleolus, metatarsal head 1 and 5 and both heels. Tracking markers clusters were firmly secured to the anterolateral aspect of both thighs (8) and shanks (8). All trials were visually inspected using Visual3D v5 software (C-Motion, Germantown, Canada). Kinematic data were filtered using a lowpass Butterworth filter with a cutoff frequency set at $6 \mathrm{~Hz}$. Gait events such as toe off (TO) and initial contact (IC) were detected using previously validated coordinate- and marker-based algorithms respectively $[11,13]$. Sagittal and kinematics of the hip, knee and ankle joint were time normalized and averaged across trials for each subject, with a gait cycle starting and ending at initial contact of the observed side. Additionally, the following spatiotemporal variables were computed for each subject separately: speed, stride length, cadence, swing time, stance time and cycle time. 


\subsection{Statistical analysis}

A one-way ANOVA between-subjects analysis was used to identify statistically significant differences between groups for knee range of motion and corrected muscle strength values. Similarly, peak angles and time at which the peak angle occurred for hip, knee and ankle joint during stance and swing were compared between groups. All statistical tests were conducted in SPSS (version 24, IBM corp, Armonk, NY).

Statistical parametric mapping (SPM) [16] was used to assess statistically significant differences between groups for gait kinematics. Specifically, a one-way between subjects ANOVA was used to compare sagittal plane kinematics of the hip, knee and ankle joint in the three groups (both arthroplasty groups and the control group). The scalar output SPM (F) was calculated for each time node separately and in order to test the null hypothesis, a critical threshold was calculated at which only $\alpha=0.05$ of smooth random curves would be expected to traverse. This threshold was based upon estimates of trajectory smoothness via temporal gradients [16], and Random Field Theory expectations regarding the field wide maximum [1]. Adjacent points exceeding this threshold are called "supra-threshold clusters" for which specific p-values were calculated using Random Field Theory, indicating the probability with which the supra-threshold clusters would be produced by a random field process with the same temporal smoothness. All SPM analyses were implemented using open-source spm1d code in Matlab (R2016b, 8.6.0, Matworks Inc, Natick, MA).

\section{Results}

\subsection{Clinical examination}

Findings from the clinical examination are presented in Table 2. No significant differences could be demonstrated when comparing knee flexion RoM between the operated leg (OPL) of UKA and TKA subjects. However, both UKA and TKA showed significantly less knee flexion at the OPL than the control group. UKA subjects also showed significantly less knee extension compared to the healthy control group. Knee replacement subjects showed significantly less strength of the gluteus medius at the OPL and at the non-operated leg (NOPL) compared to control subjects. Furthermore, TKA subjects also demonstrated weaker quadriceps and hamstrings strength at both legs compared to healthy controls, whereas UKA subjects only displayed bilateral weakness of the hamstrings compared to the control group. Patients with TKA showed less strength of the gluteus maximus at the OPL compared to healthy subjects. No differences in muscle strength and knee range of motion could be demonstrated between UKA and TKA subjects.

\subsection{Gait analysis}

\subsubsection{SPM-analysis}

The results from the SPM analysis are presented in Fig. 1. Statistically significant differences were found for the sagittal knee joint kinematics. Hip flexion and extension profiles were similar in the three groups. Identically smooth random 1D data would produce a cluster of this breath with a probability of $P=0.042$. Furthermore, UKA subjects showed significantly more everted ankle than healthy and TKA subjects at $30-50 \%$ of the gait cycle. Ankle dorsi- and plantarflexion were similar in the three groups. Identically smooth random 1D data would produce a cluster of this breath with a probability of $P=0.002$. Both TKA and UKA subjects showed significantly less knee flexion at 59-70\% of the gait cycle compared to healthy controls.

\subsubsection{Peak angles and timing analysis}

The results from the peak angle analysis are presented in Tables 3 and 4 and demonstrate additional results to the SPM-analysis. For instance, TKA subjects demonstrated significantly less peak knee flexion than healthy controls during the loading phase and midstance $(P<0.001)$. During swing, both UKA and TKA subjects demonstrated less peak ankle plantarflexion than the healthy control group $(P=0.03)$. The timing upon which maximal and minimal joint angles were reached during stance and swing was found to be significantly different between groups. UKA subjects reached maximal hip extension at $46.2 \%$ of the gait cycle whereas subjects with TKA only reached it at $50.5 \%$ of the gait cycle. Similarly to the hip joint, TKA subjects reached a maximal ankle plantarflexion during stance sooner than healthy controls, at $24.4 \%$ of the gait cycle, vs. $59.90 \%$ of the gait cycle in healthy controls.

\subsubsection{Spatiotemporal analysis}

The spatiotemporal gait parameters of the three groups are presented in Table 5 . No differences were demonstrated between groups for stance, swing and cycle time duration. However, TKA subjects demonstrated a significantly shorter stride length $(P=0.05)$ and slower speed $(P=0.04)$ than healthy controls.

\section{Discussion}

To our knowledge, this is the first study reporting arthroplastyspecific differences between patients with UKA and TKA regarding sagittal plane kinematics of the entire lower limb at the OPL during gait.

Patients with UKA and TKA showed significantly less knee flexion during the swing phase. Additionally, peak knee flexion angles at different time points revealed that TKA subjects walked with reduced knee flexion angles during loading response and midstance. Although this finding was not supported by an SPM analysis, similar findings have

Table 2

Physical examination.

\begin{tabular}{|c|c|c|c|c|c|c|c|}
\hline & \multicolumn{2}{|l|}{ UKA } & \multicolumn{2}{|l|}{ TKA } & \multicolumn{2}{|l|}{ Healthy } & \multirow[t]{2}{*}{$P$} \\
\hline & Mean & SD & Mean & SD & Mean & SD & \\
\hline Knee flexion OPL $\left(^{\circ}\right)$ & 122.67 & 5.24 & 127.13 & 7.92 & 140.67 & 5.65 & $<0.01^{1,2}$ \\
\hline Knee extension OPL $\left({ }^{\circ}\right)$ & -2.83 & 2.79 & -0.50 & 1.41 & 0.00 & 0.00 & $0.03^{1}$ \\
\hline Gluteus Medius OPL (N/m) & 151.65 & 37.58 & 153.65 & 27.93 & 272.56 & 32.14 & $<0.01^{1,2}$ \\
\hline Gluteus Medius NOPL (N/m) & 165.53 & 52.95 & 148.48 & 32.33 & 283.09 & 28.29 & $0.01^{1,2}$ \\
\hline Gluteus Maximus OPL (N/m) & 134.61 & 35.67 & 123.41 & 32.17 & 185.46 & 44.53 & $0.02^{1}$ \\
\hline Gluteus Maximus NOPL (N/m) & 139.51 & 52.63 & 122.01 & 55.49 & 171.72 & 42.33 & n.s. \\
\hline Hamstrings OPL $(\mathrm{N} / \mathrm{m})$ & 103.91 & 29.65 & 84.60 & 25.07 & 180.04 & 22.00 & $<0.01^{1,2}$ \\
\hline Hamstrings NOPL $(\mathrm{N} / \mathrm{m})$ & 98.00 & 20.10 & 89.55 & 30.43 & 187.67 & 22.85 & $<0.01^{2}$ \\
\hline Quadriceps OPL (N/m) & 204.53 & 67.06 & 162.51 & 38.24 & 225.07 & 41.49 & $<0.01^{1}$ \\
\hline Quadriceps NOPL (N/m) & 197.20 & 73.11 & 186.22 & 48.37 & 275.63 & 32.92 & $0.02^{1}$ \\
\hline
\end{tabular}

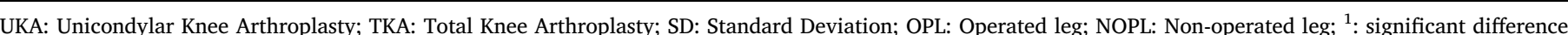
between UKA and Healthy; ${ }^{2}$ : significant difference between TKA and Healthy, ${ }^{3}$ significant difference between UKA and TKA. 

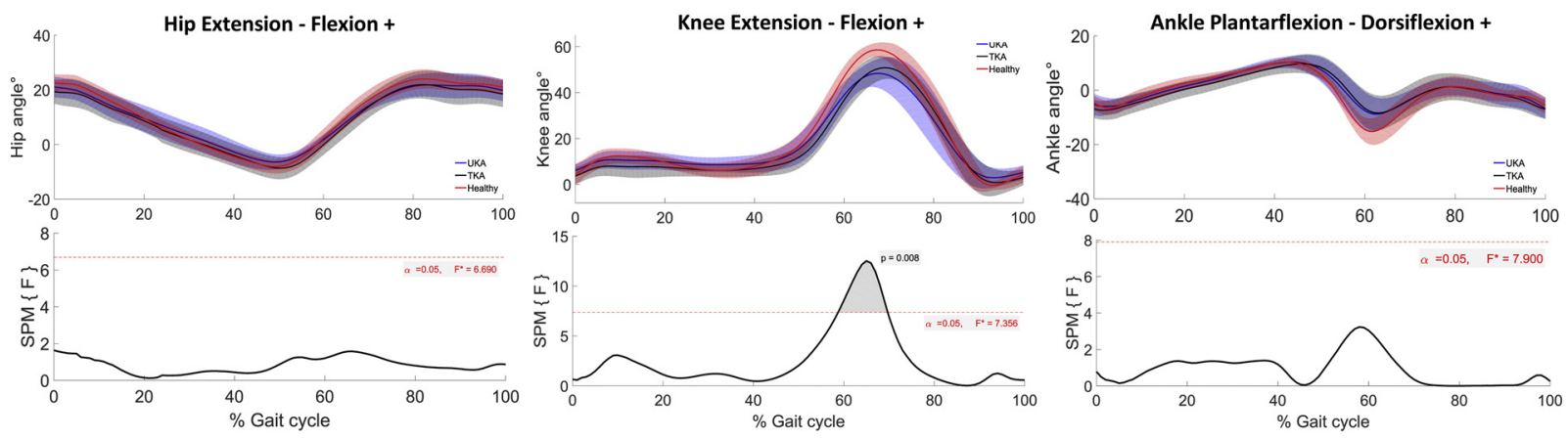

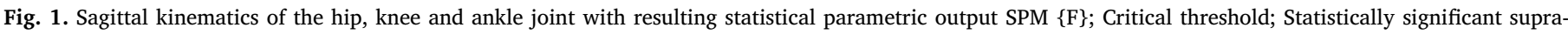
threshold cluster.

Table 3

Spatiotemporal gait parameters.

\begin{tabular}{lllll}
\hline & $\begin{array}{l}\text { UKA } \\
\text { Mean ( } \pm \text { SD) }\end{array}$ & $\begin{array}{l}\text { TKA } \\
\text { Mean }( \pm \text { SD) }\end{array}$ & $\begin{array}{l}\text { Healthy } \\
\text { Mean ( } \pm \text { SD) }\end{array}$ & P \\
\hline Cycle time (s) & $1.24(0.15)$ & $1.30(0.11)$ & $1.23(0.16)$ & n.s. \\
Stance time (s) & $0.73(0.08)$ & $0.73(0.07)$ & $0.73(0.09)$ & n.s. \\
Swing time (s) & $0.51(0.90)$ & $0.51(0.04)$ & $0.50(0.07)$ & n.s. \\
Cadans (steps/minute) & $99.25(12.59)$ & $93.96(9.47)$ & $100.21(15.78)$ & n.s. \\
Speed (m/s) & $0.90(0.18)$ & $0.75(0.10)$ & $0.96(0.17)$ & $\mathbf{0 . 0 4}^{2}$ \\
Stride width (cm) & $14.9(3.53)$ & $14.0(2.30)$ & $12.4(3.12)$ & n.s. \\
Stride length (cm) & $1.09(17.31)$ & $0.98(12.90)$ & $1.16(5.40)$ & $\mathbf{0 . 0 5}^{2}$
\end{tabular}

UKA: Unicondylar Knee Arthroplasty; TKA: Total Knee Arthroplasty; SD: Standard Deviation, ${ }^{1}$ : significant difference between UKA and Healthy; ${ }^{2}$ : significant difference between TKA and Healthy, ${ }^{3}$ significant difference between UKA and TKA.

been reported in previous studies performed on TKA subjects $[14,26,34]$. Noteworthy is that the reduced knee flexion angles during stance could not be related to a reduced knee flexion RoM, as TKA subjects showed significantly higher knee flexion at the OPL. Furthermore, a decrease in knee flexion angles during stance was identified as a crucial contributor in unloading the quadriceps, as it reduces the external knee flexion moment [28]. Berchuck et al. described this gait pattern as "quadriceps avoidance gait". Several factors such as pain, abnormal knee joint function, quadriceps weakness and proprioceptive impairments have been associated with a reduced knee range of motion during stance [34]. In the present study, TKA patients showed significantly less quadriceps strength at the OPL compared to healthy subjects, which in turn can be related to the observed quadriceps avoidance gait even though no significant differences in quadriceps strength or pain scores could be observed between UKA and TKA subjects. Remarkably, UKA patients did not display any kinematical adaptation that might relate to quadriceps avoidance, on the contrary, normal peak knee flexion values were observed during the loading response. These findings might indicate that quadriceps weakness is not the main contributor to quadriceps avoidance gait in the knee arthroplasty population. Noteworthy is that muscle force was assessed by means of a break test, which evaluates a subjects' maximal voluntary isometric contraction in a predefined and standardized position. The execution of a dynamic task such as gait will require, besides sufficient muscle force, an optimal neuromuscular control. Possibly a much more important contributing factor might be the altered proprioceptive information arising from the knee joint, due to absence of the cruciate ligaments $[28,40]$. However, further research is necessary is to support this statement. Additionally, previous researchers demonstrated that due to a quadriceps avoidance gait pattern, TKA patients showed an

Table 4

Sagittal peak angles and timing of sagittal peak angles during stance.

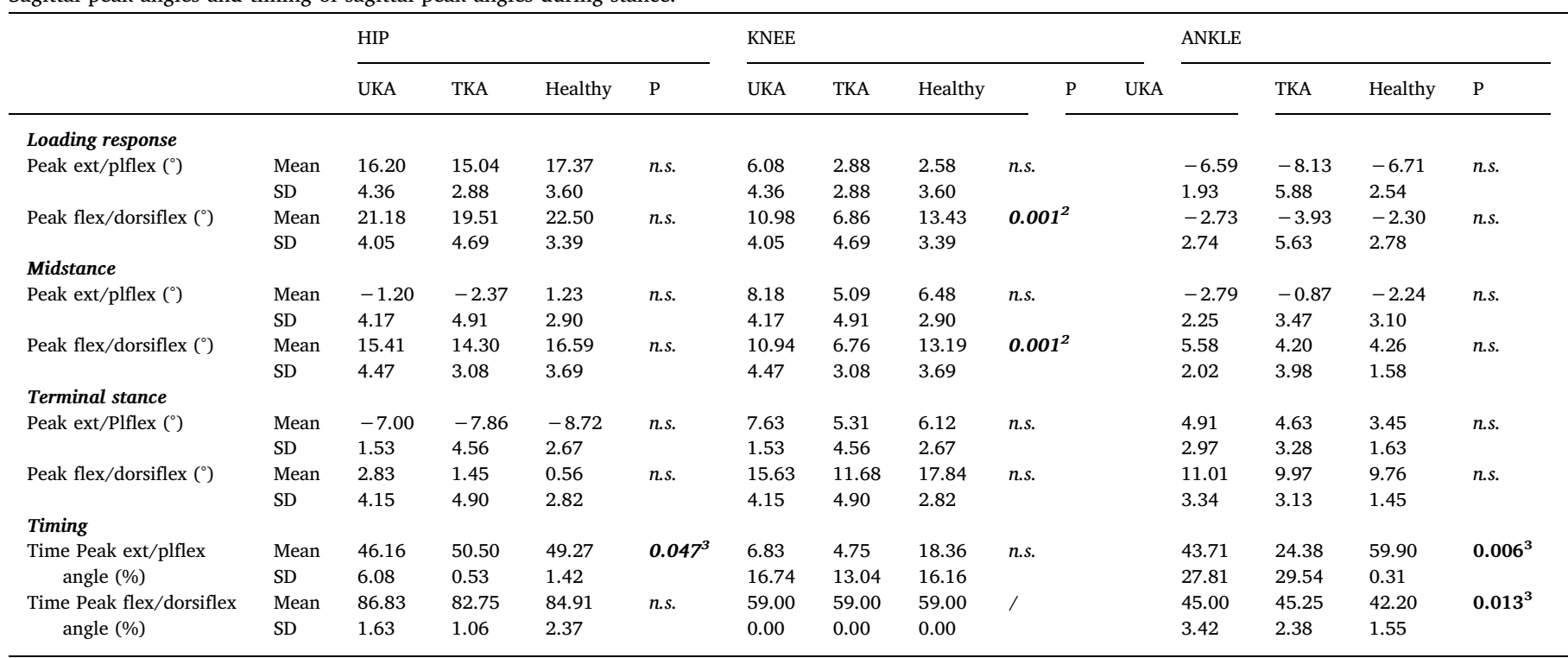

UKA: Unicondylar Knee Arthroplasty; TKA: Total Knee Arthroplasty; SD: Standard Deviation; ext: exten.s.ion; flex: flexion; plflex: plantarflexion; dorsiflex: dorsiflexion; abd: abduction; add: adduction; valg: valgus; var: varus; ev: eversion; inv: inversion; ${ }^{1}$ : significant difference between UKA and Healthy; ${ }^{2}$ : significant difference between TKA and Healthy, ${ }^{3}$ significant difference between UKA and TKA. 
Table 5

Sagittal peak angles and timing of peak sagittal angles during swing.

\begin{tabular}{|c|c|c|c|c|c|c|c|c|c|c|c|c|c|}
\hline & & \multicolumn{4}{|l|}{ HIP } & \multicolumn{4}{|l|}{ KNEE } & \multicolumn{4}{|l|}{ ANKLE } \\
\hline & & UKA & TKA & Healthy & $\mathrm{P}$ & UKA & TKA & Healthy & $\mathrm{P}$ & UKA & TKA & Healthy & $\mathrm{P}$ \\
\hline \multicolumn{14}{|l|}{ Swing } \\
\hline \multirow[t]{2}{*}{ Peak ext/plflex $\left({ }^{\circ}\right.$ ) } & Mean & -0.38 & -3.42 & 1.75 & n.s. & 2.17 & 0.13 & -2.19 & n.s. & -11.41 & -10.26 & -16.28 & $0.03^{2}$ \\
\hline & SD & 2.06 & 3.77 & 3.73 & & 2.58 & 3.91 & 3.41 & & 4.90 & 5.00 & 5.06 & \\
\hline \multirow[t]{2}{*}{ Peak flex/dorsiflex $\left({ }^{\circ}\right)$} & Mean & 22.26 & 17.31 & 21.99 & n.s. & 49.82 & 47.56 & 59.87 & $0.001^{1,2}$ & 2.19 & 2.00 & 1.59 & n.s. \\
\hline & SD & 4.78 & 5.36 & 3.14 & & 6.72 & 6.24 & 3.78 & & 3.40 & 5.46 & 3.69 & \\
\hline \multicolumn{14}{|l|}{ Timing } \\
\hline \multirow{2}{*}{$\begin{array}{l}\text { Time Peak ext/plflex } \\
\text { angle }(\%)\end{array}$} & Mean & 60.00 & 60.00 & 60.00 & / & 92.33 & 94.00 & 93.00 & n.s. & 73.14 & 72.75 & 61.20 & n.s. \\
\hline & SD & 0.00 & 0.00 & 0.00 & & 3.14 & 3.34 & 2.68 & & 18.41 & 16.61 & 0.79 & \\
\hline \multirow{2}{*}{$\begin{array}{l}\text { Time Peak flex/dorsiflex } \\
\text { angle (\%) }\end{array}$} & Mean & 0.67 & 0.38 & 1.27 & n.s. & 67.83 & 69.00 & 67.45 & n.s. & 79.29 & 81.13 & 80.60 & n.s. \\
\hline & SD & 5.69 & 2.38 & 6.38 & & 2.79 & 2.14 & 2.07 & & 4.96 & 3.04 & 4.40 & \\
\hline
\end{tabular}

increased hip extensor demand in order to have a functional locomotion [31]. In order to cope with this increased demand, one would expect higher gluteus maximus and hamstrings strength values [2]. However, in the present study, significantly lower gluteus maximus and hamstrings strength values were observed in the TKA group compared to healthy control subjects. Furthermore, recruiting the gluteus maximus to create a forward momentum will not result in an efficient locomotion as the primary role of this muscle is to provide support to the center of mass and breaking during gait, rather than propulsion [39]. Based on the findings presented in this study and evidence reported for other severe knee pathologies (e.g. anterior cruciate ligament injury) [18], one may suggest that knee replacement patients will benefit from a specific gait-retraining protocol which also includes an exercise program addressing muscles (including the hip) of the entire lower limb.

When interpreting the results of this study, some limitations should be acknowledged. Most importantly, the small sample size as a consequence of the strict inclusion and exclusion criteria makes that caution should be taken to generalizing the results. The present dataset is nonetheless unique, as it contains data from highly matched subjects. A significantly lower body mass index was observed among subjects in the control group compared to those with a knee replacement. Sagittal plane kinematics should therefore also be interpreted with caution, since a higher body mass index could cause soft tissue artefacts [9]. Nevertheless, an increased BMI is a very common problem among patients with a knee replacement [19]. Yet, at this point there are no noninvasive alternatives to 3D gait analysis as far as we know. Finally, the male/female distribution might also have affected the resulting sagittal kinematics [8]. Unfortunately, the male/female distribution and high BMI are inherent characteristics in the knee replacement population, and would therefore be difficult limitations to overcome even in future studies with larger population samples.

\section{Conclusion}

Both TKA and UKA patients showed arthroplasty-specific gait adaptations in the sagittal plane and muscle weakness of the quadriceps and hip stabilizers one year post knee replacement surgery. Yet, kinematical patterns of a quadriceps avoidance gait were only observed in the TKA group. These arthroplasty specific adaptations after knee replacement surgery should be taken into account when planning postsurgery follow-up and rehabilitation.

UKA: Unicondylar Knee Arthroplasty; TKA: Total Knee Arthroplasty; SD: Standard Deviation; ext: extension; flex: flexion; plflex: plantarflexion; dorsiflex: dorsiflexion; abd: abduction; add: adduction; valg: valgus; var: varus; ev: eversion; inv: inversion; ${ }^{1}$ : significant difference between UKA and Healthy; ${ }^{2}$ : significant difference between TKA and Healthy, ${ }^{3}$ significant difference between UKA and TKA

\section{Declaration of Competing Interest}

There are no conflicts of interest in this work.

\section{Acknowledgements}

This research was funded by the European Regional Development Fund (We-Lab for Health, Technology and Movement, project number 1047).

\section{References}

[1] R.J. Adler, Random Fields and Geometry, Springer New York, New York, NY, 2007.

[2] M.M. Ardestani, M. Moazen, How human gait responds to muscle impairment in total knee arthroplasty patients: muscular compensations and articular perturbations, J. Biomech. 49 (2018) 1620-1633.

[3] A. Arirachakaran, P. Choowit, C. Putananon, S. Muangsiri, J. Kongtharvonskul, Is unicompartmental knee arthroplasty (UKA) superior to total knee arthroplasty (TKA)? A systematic review and meta-analysis of randomized controlled trial, Eur. J. Orthop. Surg. Traumatol. 25 (2018) 799-806.

[4] J.B. Arnold, S. Mackintosh, T.S. Olds, S. Jones, D. Thewlis, Improvements in knee biomechanics during walking are associated with increased physical activity after total knee arthroplasty, J. Orthop. Res. 33 (2015) 1818-1825.

[5] R. Bourne, B. Chesworth, A. Davis, N. Mahomed, K. Charron, Patient satisfaction after total knee arthroplasty: who is satisfied and who is not? Clin. Orthop. Relat. Res. 468 (2010) 57-63.

[6] L. Brosseau, M. Tousignant, J. Budd, N. Chartier, L. Duciaume, S. Plamondon, et al. Intratester and intertester reliability and criterion validity of the parallelogram and universal goniometers for active knee flexion in healthy subjects, Physiother. Res. Int. 2 (1997) 150-166.

[7] N.M. Brown, N.P. Sheth, K. Davis, M.E. Berend, A.V. Lombardi, K.R. Berend, et al., Total knee arthroplasty has higher postoperative morbidity than unicompartmental knee arthroplasty: a multicenter analysis, J. Arthroplasty 27 (2012) 86-90.

[8] D.A. Bruening, R.E. Frimenko, C.D. Goodyear, D.R. Bowden, A.M. Fullenkamp, Sex differences in whole body gait kinematics at preferred speeds, Gait Posture 41 (2015) 540-545.

[9] B. Callewaert, L. Labey, A. Leardini, J. Bellemans, K. Desloovere, L. Scheys, High versus normal body- mass index: effects on 3D kinematics and kinetics during dailylife motor tasks, Gait Posture 38 (2013) S111.

[10] V. Choksi, V. Chainani, S. Shaharyar, K. Dave, S. Ravindranathan, R. Hanno, et al., Objective measures of the frailty syndrome (hand grip strength and gait speed) and cardiovascular mortality: a systematic review, Int. J. Cardiol. 215 (2016) 487-493.

[11] K.M. Crossley, W.-J. Zhang, A.G. Schache, A. Bryant, S.M. Cowan, Performance on the single- leg squat task indicates hip abductor muscle function, Am. J. Sports Med. 39 (2011) 866.

[12] J. Dawson, R. Fitzpatrick, D. Murray, A. Carr, Questionnaire on the perceptions of patients about total knee replacement, J. Bone Joint Surg. Br. 80 (1998) 63-69.

[13] A.R. De Asha, M.A. Robinson, G.J. Barton, A marker based kinematic method of identifying initial contact during gait suitable for use in real-time visual feedback applications, Gait Posture 36 (2012) 650-652.

[14] D.A. Dennis, R.D. Komistek, J.B. Stiehl, S.A. Walker, K.N. Dennis, Range of motion after total knee arthroplasty the effect of implant design and weight-bearing conditions, J. Arthroplasty 13 (1998) 748-752.

[15] S.J. Farquhar, D.S. Reisman, L. Snyder-Mackler, Persistence of altered movement patterns during a sit-to- stand task 1 year following unilateral total knee arthroplasty, Phys. Ther. 88 (567) (2008).

[16] K.J. Friston, J.T. Ashburner, S.J. Kiebel, T.E. Nichols, W.D. Penny, Statistical Parametric Mapping: The Analysis of Functional Brain Images, Amsterdam: Elsevier, Amsterdam, 2007.

[17] S. Graves, D. Davidson, L. Ingerson, P. Ryan, E. Griffith, B. McDermott, et al., The Australian orthopaedic association national joint replacement registry, Med. J. Aust. 180 (2004) S31-S34. 
[18] M. Grodski, R. Marks, Exercises following anterior cruciate ligament reconstructive surgery: biomechanical considerations and efficacy of current approaches, Res. Sports Med. 16 (2008) 75-96.

[19] D. Guenther, S. Schmidl, T.O. Klatte, H.K. Widhalm, M. Omar, C. Krettek, et al., Overweight and obesity in hip and knee arthroplasty: evaluation of 6078 cases, World J. Orthop. 6 (2015) 137-144.

[20] G.L. Hatfield, C.L. Hubley-Kozey, J.L. Astephen Wilson, M.J. Dunbar, The effect of total knee arthroplasty on knee joint kinematics and kinetics during gait, J. Arthroplasty 26 (2011) 309-318.

[21] G.L. Hatfield, W.D. Stanish, C.L. Hubley-Kozey, Three-dimensional biomechanical gait characteristics at baseline are associated with progression to total knee arthroplasty, Arthritis Care Res. 67 (2015) 1004-1014.

[22] Y. Ivanenko, G. Cappellini, N. Dominici, R. Poppele, F. Lacquaniti, Modular control of limb movements during human locomotion, J. Neurosci. 27 (2018) 11149-11161.

[23] D.A. Krause, S.J. Schlagel, B.M. Stember, J.E. Zoetewey, J.H. Hollman, Influence of lever arm and stabilization on measures of hip abduction and adduction torque obtained by hand- held dynamometry, Arch. Phys. Med. Rehabil. 88 (2007) 37-42.

[24] S.H. Lastad Lygre, B. Espehaug, L.I. Havelin, O. Furnes, S.E. Vollset, Pain and function in patients after primary unicompartmental and total knee arthroplasty, J. Bone Jt. Surg. 92 (2018) 2890.

[25] J. Lewis, G. Freisinger, X. Pan, R. Siston, L. Schmitt, A. Chaudhari, Changes in lower extremity peak angles, moments and muscle activations during stair climbing at different speeds, J. Electromyogr. Kinesiol. 25 (2015) 982-989.

[26] K. Li, D.C. Ackland, J.A. McClelland, K.E. Webster, J.A. Feller, R. de Steiger, et al., Trunk muscle action compensates for reduced quadriceps force during walking after total knee arthroplasty, Gait Posture 38 (2013) 79-85.

[27] U.G. Longo, M. Loppini, U. Trovato, G. Rizzello, N. Maffulli, V. Denaro, No difference between unicompartmental versus total knee arthroplasty for the management of medial osteoarthtritis of the knee in the same patient: a systematic review and pooling data analysis, Br. Med. Bull. 114 (2015) 65-73.

[28] M. Berchuck, T.P. Andriacchi, B.R. Bach, B. Reider, Gait adaptations by patients who have a deficient anterior cruciate ligament, Clin. J. Sport. Med. 1 (1990) 214.

[29] J.A. Feller, J.A. McClelland, K.E. Webster, Gait analysis of patients following total knee replacement: a systematic review, Knee 14 (2007) 253-263.

[30] J.A. McClelland, J.E. Wittwer, K.E. Webster, J.A. Feller, Long-term assessment of gait biomechanics in patients with total knee arthroplasty, Osteoarthr. Cartil. 222014 (2014) S90.
[31] C.A. McGibbon, D.E. Krebs, Compensatory gait mechanics in patients with unilateral knee arthritis, J. Rheumatol. 29 (2002) 2410.

[32] B. Mentiplay, L. Perraton, K. Bower, B. Adair, Y.-H. Pua, G. Williams, et al., Assessment of lower limb muscle strength and power using hand- held and fixed dynamometry: a reliability and validity study, PLoS One 10 (2015).

[33] C. Milner, Is gait normal after total knee arthroplasty? Systematic review of the literature, J. Orthop. Sci. 14 (2009) 114-120.

[34] R.L. Mizner, L. Snyder-Mackler, Altered loading during walking and sit-to-stand is affected by quadriceps weakness after total knee arthroplasty, J. Orthop. Res. 23 (2005) 1083-1090.

[35] C. Pabinger, H. Lothaller, A. Geissler, Utilization rates of knee-arthroplasty in OECD countries, Osteoarthr. Cartil. 23 (2015) 1664-1673.

[36] T.C. Pataky, M.A. Robinson, J. Vanrenterghem, Vector field statistical analysis of kinematic and force trajectories, J. Biomech. 46 (2013) 2394-2401.

[37] K.D. Plancher, S.K. Bishai, D.G. Areson, Predictors of patient satisfaction following unicondylar knee arthroplasty: 1153, Med. Sci. Sports Exerc. 40 (2008) S144.

[38] Y.H. Pua, F.J. Seah, R.A. Clark, C. Lian-Li Poon, J.W. Tan, H.C. Chong, Factors associated with gait speed recovery after total knee arthroplasty: a longitudinal study, Semin. Arthritis Rheum. (2016).

[39] S. Schloemer, J. Thompson, A. Silder, D. Thelen, R. Siston, Age- related differences in gait kinematics, kinetics, and muscle function: a principal component analysis, J. Biomed. Eng. Soc. 45 (2017) 695-710.

[40] M.R. Torry, M.J. Decker, R.W. Viola, D.D. O'connor, J. Richard Steadman, Intraarticular knee joint effusion induces quadriceps avoidance gait patterns 11 , Presented at the XVII Congress of the International Society of Biomechanics (1999) Clinical Biomechanics 15:147-159.

[41] K. Turcot, Y. Sagawa Jr, D. Fritschy, P. Hoffmeyer, D. Suva, S. Armand, How gait and clinical outcomes contribute to patients' satisfaction three months following a total knee arthroplasty, J. Arthroplasty 28 (2013) 1297-1300.

[42] J. Vanrenterghem, D. Gormley, M. Robinson, A. Lees, Solutions for representing the whole-body centre of mass in side cutting manoeuvres based on data that is typically available for lower limb kinematics, Gait Posture 31 (2010) 517-521.

[43] K.E. Webster, J.E. Wittwer, J.A. Feller, Quantitative gait analysis after medial unicompartmental knee arthroplasty for osteoarthritis, J. Arthroplasty 18 (2003) 751-759.

[44] H.A. Wilson, R. Middleton, S.G.F. Abram, S. Smith, A. Alvand, W.F. Jackson, et al. Patient relevant outcomes of unicompartmental versus total knee replacement: systematic review and meta-analysis, BMJ (Clin. Res. Ed.) 364 (2019) 1352. 